\title{
Avaliações antioxidante e antifúngica dos óleos essenciais de Hymenaea stigonocarpa Mart. ex Hayne e Hymenaea courbaril L.
}

\author{
Antonio Carlos Pereira de Menezes Filho ${ }^{\mathrm{a}^{*}}{ }^{\oplus}$, Josemar Gonçalves de Oliveira Filho ${ }^{\mathrm{b}}{ }^{\circledR}$, \\ Carlos Frederico de Souza Castro ${ }^{a}$ \\ ${ }^{\text {a }}$ Instituto Federal Goiano, Brasil \\ ${ }^{\mathrm{b}}$ Universidade Estadual Júlio de Mesquita Filho, Brasil \\ *Autor correspondente (astronomoamadorgoias@gmail.com)
}

\section{N F O}

\section{Keywords}

biological activity

volatile oil

fruit shell

DPPH

Sclerotinia

Aspergillus

Colletotrichum

\section{Palavras-chaves} atividade biológica óleo volátil casca fruto DPPH

Sclerotinia Aspergillus Colletotrichum

\begin{abstract}
A B S T R A C T
Antifungal and antioxidant evaluations of the essential oils from Hymenaea stigonocarpa Mart. ex Hayne and Hymenaea courbaril L.

Hymenaea stigonocarpa and Hymenaea courbaril var. courbaril are plant species belonging to the Leguminosae family, found in the Cerrado domain. They present annual fruiting with fruits with dense and aromatic peel. The objective of this work was to evaluate the yield, antioxidant and antifungal activities of pure oil and mix (mixture of both oils in equal concentration). For extraction, Clevenger type equipment was used, the yield was determined in percentage. The antioxidant activity was determined in microplates using the DPPH radical as a model in the following concentrations, starting from pure oil, $50,40,30,20,10,5,2.5$ and $1.25 \mu \mathrm{LL}^{-1}$. The antifungal activity was evaluated by agar diffusion using successive dilutions of pure essential oil and in concentrations of 50, 25, 12.5, $6.25,3.13$ and $1.56 \mu \mathrm{L} \mathrm{mL}^{-1}$ and mix mixture of both oils in equal parts in DMSO, on Sclerotinia sclerotiorum, Colletotrichum acutatum and gloeosporioides, and for Aspergillus flavus and niger. The yield was 0.04 and $0.06 \%$ for $H$. stigonocarpa and $H$. courbaril, respectively. The antioxidant activity showed good results, for both essential oils of $H$. stigonocarpa and $H$. courbaril with high reduction of DPPH radical to pure and in concentrations 50 and $40 \mu \mathrm{L} \mathrm{mL}^{-1}$. For the mix, the concentration is $50 \mu \mathrm{L} \mathrm{mL}^{-1}$, a rate of $72 \%$ reduction. In the antifungal experiment, high efficiency was observed in the growth control of hyphae for the essential oil of $H$. stigonocarpa with $83 \%$ on Sclerotinia sclerotiorum, $75 \%$ on C. acutatum, and for A. flavus with $55 \%$, respectively. For $H$. courbaril essential oil, an inhibition rate of $100 \%$ was observed for S. sclerotiorum, $100 \%$ for C. gloeosporioides and $70 \%$ for A. flavus. The essential oils mix showed a reduction in growth of $88 \%$ for $S$. sclerotiorum, $56 \%$ for C. gloesporioides and $42 \%$ for A. niger. Pure and mix essential oils showed promising results as natural antioxidant and antifungal agents in vitro.
\end{abstract}

\section{R E S U M O}

Hymenaea stigonocarpa e Hymenaea courbaril var. courbaril são espécies vegetais pertencentes a família Leguminosae, sendo encontrados no domínio Cerrado. Apresentam frutificação anual com frutos com casca densa e aromática. O trabalho teve por objetivo avaliar o rendimento, e as atividades antioxidante e antifúngica do óleo puro e mix (mistura de ambos os óleos em igual concentração). Para extração, foi utilizado equipamento tipo Clevenger, o rendimento foi determinado em porcentagem. A atividade antioxidante foi determinada em microplacas utilizando como modelo o radical DPPH nas seguintes concentrações, partindo do óleo puro, 50, 40, 30, 20, 10, 5, 2,5 e 1,25 $\mu \mathrm{L}$ $\mathrm{mL}^{-1}$. A atividade antifúngica foi avaliada por difusão em ágar utilizando sucessivas diluições de óleo essencial puro e nas concentrações $50,25,12,5,6,25,3,13$ e $1,56 \mu \mathrm{L} \mathrm{mL}^{-1}$ e mix mistura de ambos os óleos em partes iguais em DMSO, sobre Sclerotinia sclerotiorum, Colletotrichum acutatum e gloeosporioides, e para Aspergillus flavus e niger. $\mathrm{O}$ rendimento foi de 0,04 e $0,06 \%$ para $H$. stigonocarpa e $H$. courbaril, respectivamente. A atividade antioxidante apresentou bons resultados, para ambos os óleos essenciais de $H$. stigonocarpa e $H$. courbaril com alta redução do radical DPPH para puro e nas concentrações 50 e $40 \mu \mathrm{L} \mathrm{mL} \mathrm{mL}^{-1}$. Já para o mix a concentração $50 \mu \mathrm{L} \mathrm{mL}^{-1}$ taxa de $72 \%$ de redução. No experimento antifúngico foi observada alta eficiência no controle de crescimento das hifas para o óleo essencial de $H$. stigonocarpa com $83 \%$ sobre Sclerotinia sclerotiorum, $75 \%$ sobre C. acutatum, e para A. flavus com $55 \%$, respectivamente. Para o óleo essencial de $H$. courbaril foi observada taxa de inibição de $100 \%$ para $S$. sclerotiorum, $100 \%$ para C. gloeosporioides e de $70 \%$ para A. flavus. Já para o mix de óleos essenciais foi observada redução no crecimento de $88 \%$ para S. sclerotiorum, $56 \%$ C. gloesporioides e de $42 \%$ para A. niger. Os óleos essenciais puros e mix apresentaram resultados promissores como agentes antioxidantes e antifúngicos naturais in vitro. 


\section{INTRODUÇÃO}

A família Leguminosae inclui aproximadamente 727 gêneros e mais de 19.327 espécies, estando presente em todos os continentes, exceto na Antártida. No Brasil são registrados 221 gêneros e mais de 2.809 espécies, sendo 156 gêneros e 925 espécies (Souza et al., 2016). Conforme Souza et al. (2016), De Candolle (1825), posicionou Hymenaea na tribo Cassieae (Caesalpinioideae), entretanto, estudos posteriores consideram o gênero inserido em Detarieae (Mackinder, 2005). Estudos genéticos e de sistemática sustentam o monofiletismo da tribo Detarieae, sendo o clado Hymenaea apresentando estreita afinidade entre os gêneros Hymenaea, Guibourtia Benn. e Peltogyne Vogel.

$\mathrm{O}$ gênero Hymenaea, tem entre diversas espécies, os indivíduos Hymenaea stigonocarpa Mart. ex Hayne conhecido por "jatobá-docerrado" e o Hymenaea courbaril L. conhecido popularmente por "jatobá-do-campo". Lee e Langenheim (1975) propuseram em revisão do gênero o reconhecimento de duas seções: $1^{a}$ Hymenaea apresentando características nas inflorescências curto-paniculadas, com $8-15 \mathrm{~cm}$ compr., corimbosas quando maduras, e flores de tamanho médio; e $2^{\mathrm{a}}$ Trachylobium (Hayne) Baill., por inflorescências longo-paniculadas, com 18-35 $\mathrm{cm}$ compr., com flores pequenas. Nos estudos de Pestana (2010), o pesquisador prôpos sinonimizações da espécie dúbia $H$. capanema a $H$. courbaril var. courbaril, e das variedades de $H$. stigonocarpa.

Hymenaea é um gênero neotropical, com distribuição disjunta na costa leste da África onde ocorre apenas uma espécie (Mackinder, 2005). Um total de 17 espécies, sendo 15 são citadas somente no Brasil, sendo utilizadas para extração de madeira, resina, casca, folhas e frutos (Lima; Pinto, 2015; Souza, 2013; Aquino et al., 2007).

Ambas as espécies são encontradas no domínio Cerrado, apresentando anualmente grandes safras de frutos que servem de alimento para roedores, veados e também para o homem do campo. Os frutos apresentam casca dura e densa, contendo nesta, resina e óleo essencial (OE) com aroma forte e característico (Menezes Filho et al., 2018; Carvalho Filho et al., 2003; Botelho et al., 2000; Silva et al., 1998).

Os óleos essenciais (OEs) são produtos do metabolismo secundário dos vegetais, sendo produzido naturalmente, estando envolvidos no processo de polinização, na defesa, agindo contra animais herbívoros, insetos e em fitopatologias as quais podem levar o vegetal à morte. São extraídos através das técnicas por arraste a vapor ou por prensagem a frio, esta última, menor difundida entre os químicos orgânicos que trabalham com produtos naturais, há outras técnicas, estas mais atuais utilizando fluído supercrítico, enfleurage, por solventes que estão sendo empregadas na extração de OEs (Bizzo et al., 2009). Os OEs são compostos principalmete por monoterpenos, diterpenos, sesquiterpenos e fenilpropanoides, esta constituição confere as características organolépticas e biológicas, ações com atividade atinfúngica e antibacteriana, dentre outras, como antioxidante, antiinflamatória, antitumoral, anti-hiperlipidêmico, imunomodulador, antinociceptivos, citotóxicos e antivirais (Fallah et al., 2020; Lima et al., 2006; Jahaniani et al., 2005; Glshani et al., 2004; Amirhofran et al., 2000; Sajjadi et al., 1998), sendo também populares entre as indústrias farmacêuticas, de alimentos, de perfumaria e de cosméticos (Himed et al., 2019).

Agentes antioxidantes naturais são compostos de classes químicas produzidas naturalmente pelas plantas através do metabolismo secundário do vegetal. Os OEs apresentam ser eficiêntes na função de proteção contra inúmeros radicais livres que constantemente são absorvidos pelo nosso organismo. A oxidação é o processo metabólico que possui a função de produzir energia para atividades enssenciais na manutenção do corpo. $\mathrm{O}$ metabolismo do $\mathrm{O}_{2}$ nas células humanas e de animais, levam a produção de radiais livres que são responsáveis por provocar danos ao organismo em nível celular, na molécula de ácido desoxirribonucléico (DNA), no tecido lipídico, em proteínas, dentre outras (Roesler et al., 2007). De acordo com Shami e Moreira (2004), dentre os radicais livres estão incluídos o superóxido $\left(\mathrm{O}_{2}{ }^{\circ}\right)$, a hidroxila $\left(\mathrm{OH}^{*}\right)$, o hidroperóxido $\left(\mathrm{HO}_{2}{ }^{\circ}\right)$, o óxido nítrico $\left(\mathrm{NO}^{\circ}\right)$ e o dióxido de nitrogênio $\left(\mathrm{NO}_{2}{ }^{\circ}\right)$. A efetiva atividade antifúngica também é observada em inúmeros OEs contra agentes fúngicos causadores de doenças em humanos, animais e em vegetais agricultáveis.

O Sclerotinia sclerotiorum conhecido por "mofo-branco ou podridão branca da haste da soja" ataca anualmente plantações de soja e feijão causando perdas de produtividade. Este fungo produz escleródios que podem ficar viáveis no solo por até 11 anos, sendo difícil sua erradicação, pois os fungicidas sintéticos não demonstram serem eficientes na eliminação desta forma 
reprodutiva (Silva et al., 2018; Garcia et al., 2012). Este é um fungo terrícola, necrotrófico, cosmopolita e inespecífico que ataca diversas espécies vegetais em monocotiledôneas e dicotiledôneas de amplo espectro agronômico (Boland e Hall, 1994), sendo patogênico para 78 famílias, 278 gêneros e 408 espécies de plantas com importância econômica (Putrick et al., 2018).

O Colletotrichum é outro gênero fúngico que apresenta inúmeras espécies de grande importância fitopatológica na agricultura, causando diversas doenças conhecidas por antracnose. As espécies, C. gloeosporioides e $C$. acutatum causam consideráveis perdas econômicas em diversas fruticulturas ainda na fase de desenvolvimento e de pós-colheita (Ramos et al., 2016). A antracnose está presente em regiões tropicais e subtropicais, com clima propício para a fácil proliferação entre as frutas, em um curto intervalo de contaminação. Os fungos do gênero Colletotrichum se desenvolvem bem em culturas de mamão, manga, abacate, morango, pera, banana, caju, maracujá e em goiabas (Da Silva et al., 2009; Filho et al., 2003).

Menção também deve ser feita para outro gênero de fungos fitopatológicos e patológicos (Deus et al., 2011). O gênero Aspergillus, apresenta dezenas de espécies, em especial, o $A$. flavus e A. niger. Os representantes do gênero Aspergillus são considerados fungos persistentes durante o armazenamento, deteriorando principalmente grãos e sementes, produzindo a partir do seu metabolismo secundário micotoxinas e aflatoxinas (Terra, 2005; Athié et al., 1998). Esse gênero fúngico pode causar distúrbios alimentares, podendo estar ligados a diversos tipos de cânceres, bem como, no aumento das células CD4 em portadores do vírus da imunodeficiência adquirida (HIV), e também por causarem a "aspergilose" como é conhecido o grupo de doenças causadas por espécies termotolerantes de Aspergillus (Lopes et al., 2004). De acordo com Lima et al. (2014), os Aspergillus representam cerca de $10 \%$ das infecções fúngicas em seres humanos e em animais. Os principais grãos contaminados por esse gênero são amendoim, grão-de-bico, soja, milho e sorgo, bem como podem estar presentes no óleo fixo onde diminuem os valores nutricionais do produto, podendo também ocasionar óbito entre humanos e animais (De Souza et al., 2010; Dhingra e Coelho Neto, 1998; Engle et al., 1982).

$\mathrm{O}$ uso de fungicidas sintéticos apresentam efetiva atividade antifúngica, entretanto, o uso descontrolado aliado a falta de rotação de culturas, trazem sérias desvantagens para o meio ambiente, aos aplicadores e aos consumidores, além disso, ocasiona o desenvolvimento de formas resistentes de fitopatógenos (Celoto et al., 2008). A busca por alternativas mais conscientes visando a produção agrícola e a preservação do meio ambiente, a saúde humana e o bem estar animal, inúmeros trabalhos vem sendo desenvolvidos utilizando extratos vegetais, óleos fixos e OEs. E entre eles, vários apresentam importantes resultados equiparáveis aos agentes fungicidas comerciais (Hüller et al., 2019; Tico et al., 2019).

O trabalho teve por objetivo avaliar as características antioxidante e antifúngica dos óleos essenciais das cascas dos frutos de Hymenaea stigonocarpa e Hymenaea courbaril var. courbaril puro e em mix (mistura de óleos essenciais na concentração (1:1)) sobre S.sclerotiorum, Colletotrichum gloeosporioides, Colletotrichum acutatum, Aspergillus flavus e Aspergillus niger.

\section{MATERIAL E MÉTODOS}

\section{Coleta e identificação}

Os frutos de Hymenaea stigonocarpa e Hymenaea courbaril var. courbaril foram coletados no período entre março a outubro de 2019, em duas áreas de preservação permanentes (APPs), localizadas no município de Rio Verde, Goiás, Brasil. As áreas de coletas foram georreferenciadas para $H$. stigonocarpa $17^{\circ} 47^{\prime} 20.5^{\prime} \mathrm{S}$ 50 $57^{\circ} 53.9^{\prime \prime} \mathrm{W}$ e $H$. courbaril $17^{\circ} 34^{\prime} 45.1^{\prime \prime} \mathrm{S} 50^{\circ} 56^{\prime} 45.5^{\prime}$ 'W.

As espécies foram identificadas pelo Biólogo Msc. Antonio Carlos Pereira de Menezes Filho, sendo duas exsicatas herborizadas, identificadas e depositadas no Herbário do IF Goiano, com os seguintes vouchers HRV: 1.461 e 1.462.

Os frutos foram coletados manualmente na serrapilheira, e levados para o laboratório de Química Tecnológica onde foram previamente lavados com água corrente e secos a temperatura ambiente para a retirada da casca, arilo com sementes com auxílio de um martelo.

Após retirada da casca, essa foi novamente triturada com auxílio do martelo em seixos de 1 $\mathrm{cm}$ (tamanho máximo). Em seguida, o material foi triturado em moinho de facas tipo ciclone Willye (Fortinox, Mod. Star FT-60 Macro). O moinho apresenta peneira granulométrica de 32 mesh interna. O pó obtido da casca, foi armazenado em embalagem para alimentos e mantida em refrigeração a $-8{ }^{\circ} \mathrm{C}$ em freezer até a etapa de 
obtenção do OE.

\section{Extração e rendimento de óleo essencial}

Para a extração do OE, foi utilizado sistema tipo Clevenger (Marconi, Mod. MA553/2000), em refluxo por 4 horas. Para isso, três alíquotas de $100 \mathrm{~g}$ de pó foram pesadas em balança analítica digital (Marte, Mod. W220) e transferidas para balões de $2 \mathrm{~L}$ (Laborglas), acrescidos com $500 \mathrm{~mL}$ de água destilada. Após esse tempo, o hidrolato foi recolhido e transferido para funil de separação de $500 \mathrm{~mL}$ (Laborglas), onde foi lavado três vezes com $10 \mathrm{~mL}$ de diclorometano (Alphatec, P.A ACS, pureza 99,9\%). A fase orgânica foi recolhida e seca com sulfato de sódio anidro (Fmaia, P.A ACS, pureza $100 \%$ ), e em seguida, filtrado em papel de filtro qualitativo (Unifil, C42). O sobrenadante foi armazenado em frasco tipo béquer de $150 \mathrm{~mL}$ (Laborglas) envolto em papel alumínio com pequenos furos para a volatilização do solvente. $\mathrm{O}$ sistema foi mantido em ambiente climatizado com temperatura a $20^{\circ} \mathrm{C}$ até completa evaporação do solvente. Logo após, o $\mathrm{OE}$ foi pesado em balança analítica digital, e sua massa expressa em porcentagem de rendimento de extração $(\%)$.

\section{Atividade bioativa}

A atividade bioativa foi determinada conforme descrito por Mezza et al. (2018) com modificações. Utilizou-se o radical livre 2,2difenil-1-picrilhidrazil (DPPH) (Sigma-Aldrich, P.A - ACS, pureza $100 \%$ ). O ensaio antioxidante foi realizado pelo método de microdiluição em microplacas de 96 poços (Videplast), utilizando leitora de microplacas UV-Vis (Hexis Científica, Mod. Sectramax 190). Para cada poço foram adicionados $100 \mu \mathrm{L} \mathrm{mL} \mathrm{m}^{-1}$ de solução metanólica de DPPH na concentração $0,06 \mathrm{mMol} \mathrm{mL}^{-1}$, e 100 $\mu \mathrm{L} \mathrm{mL}^{-1}$ de OE diluído em metanol (Diâmica, P.A - ACS, pureza, 99,8\%) nas seguintes diluições com concentrações de 50,40,30, 20, 10, 5, 2,5 e $1,25 \mu \mathrm{L} \mathrm{mL}^{-1}$.

Neste experimento, foi avaliado a percentagem de redução do radical livre em diferentes concentrações de OE individualmente e por mix (1:1) entre os OEs de Hymenaea, como citado anteriormente. A microplaca com as amostras foi mantida em ambiente a $20^{\circ} \mathrm{C}$ na ausência de luz.
A leitura foi realizada no comprimento de ondas em $517 \mathrm{~nm}$, após $1 \mathrm{~h}$. para óleo essencial puro (OE puro), e de $35 \mathrm{~min}$. óleo essencial mix (Mix OEs) após o início da reação. Uma curva padrão foi realizada com a solução de DPPH na concentração $0,06 \mathrm{mMol}$. Como controle negativo utilizou-se a solução mãe de DPPH 0,06 mMol, e como branco o metanol. Os resultados foram expressos em percentagem de redução de DPPH (\%).

\section{Atividade antifúngica em difusão em ágar}

Para determinação da atividade antifúngica sobre os isolados de Sclerotinia slcerotiorum, Colletotrichum acutatum e Aspergillus flavus, foi realizado em diferentes concentrações OE puro, e para Sclerotinia sclerotiorum, Colletotrichum gloeosporioides e Aspergillus niger, foi utilizado mix dos OEs em ambas as espécies vegetais de Hymenaea. A metodologia em difusão em ágar foi empregada neste estudo.

Os isolados de $S$. sclerotiorum, $C$. gloeosporioides, C. acutatum, A. niger e A. flavus, foram coletados em campos de cultura de soja no município de Jataí, em fruticulturas de mamoeiro e maracujazeiros nos municípios de Itumbiara e Morrinhos, e em bagaços da cultura de cana-deaçúcar em Rio Verde, Goiás, Brasil. As culturas foram mantidas em meio batata, dextrose e ágar (Kasvi, BDA). Os isolados foram doados pelos laboratórios de Química Tecnológica e de Produtos Naturais do IF Goiano, Campus Rio Verde, Goiás, Brasil, e mantidos em banco micológico no laboratório de Química Tecnológica na mesma instituição. A atividade antifúngica dos OEs sobre o crescimento micelial dos isolados fúngicos, foi avaliado através de diferentes concentrações, partindo do $\mathrm{OE}$ puro 100 , e sucessivas diluições $50 ; 25 ; 12,5 ; 6,25 ; 3,13$ e $1,56 \mu \mathrm{L} \mathrm{mL} \mathrm{m}^{-1}$ em dimetilsulfóxido (DMSO) (Vetec, P.A - ACS, pureza 99,7\%). Como controle negativo, utilizou-se a testemunha (ausência de óleo essencial) e DMSO puro, e como controle positivo fungicida comercial Frowncide $^{\circledR} 500$ SC (ISK, Fluazinam), registro no Ministério da Aricultura $\mathrm{n}^{\circ} \mathbf{7 6 9 5}$, na concentração de $10 \mu \mathrm{L} \mathrm{mL}^{-1}$.

As concentrações dos OEs foram adicionadas ao meio de cultura BDA após esterilização e resfriamento, bem como para os tratamentos com o fungicida comercial e DMSO. Após solidificação do meio, em câmara de fluxo laminar bacteriológica (Marconi, Mod. 100 MA1550), 1 
disco de micélio para cada isolado individualmente de $S$. sclerotiorum, $C$. gloeosporioides, C. acutatum, A. flavus e A. niger com $7 \mathrm{~mm}$ de diâmetro, foi depositado no centro da placa de Petri com $10 \mathrm{~cm}$ de diâmetro. Em seguida foram incubadas em estufa bacteriológica (Nova Instruments, Mod. NI 1521) em diferentes temperaturas para cada espécie fúngica nas temperaturas $20{ }^{\circ} \mathrm{C}$ (Hamid et al., 2018), $22{ }^{\circ} \mathrm{C}$ (Bagherabadi et al., 2018), $22{ }^{\circ} \mathrm{C}$ (Bragança et al., 2016), $25{ }^{\circ} \mathrm{C}$ (Deshmukh et al., 2020), e $25{ }^{\circ} \mathrm{C}$ (Magalhães et al., 2019), respectivamente.

A avaliação consistiu em medições diárias do diâmetro das colônias, por meio de um paquímetro digital $150 \mathrm{~mm}$ (Digimes, Mod. 100-170) exatidão $0,03 \mathrm{~mm}$, resolução de $0,01 \mathrm{~mm} / .0005$ '/1/128", iniciadas 24 horas após o início da incubação e encerradas, quando as colônias fúngicas do tratamento testemunha, atingiram completamente a área interna da placa. A determinação do percentual de inibição de crescimento PIC foi realizado conforme proposto por Garcia et al. (2012). A seguir, está apresentada a equação 1, utilizada na determinação da PIC.

$$
\% \text { PIC }=(\text { DTT }- \text { DTQ }) / \text { DTT } \times 100 \text { E. [1] }
$$

Onde \%PIC $=$ percentual de inibição de crescimento, DTT = diâmetro no tratamento testemunha, DTQ = diâmetro no tratamento químico.

\section{Análise estatística}

A análise estatística consistiu em triplicatas para o rendimento de $\mathrm{OE}$ e para atividade antioxidante, e em quadruplicata para a análise antifúngica. Os resultados foram expressos através da média artimética da triplicata, seguida de $( \pm)$ desvio padrão. Os dados foram submetidos à análise de variância (ANOVA). Foi utilizado teste de Tukey $(\mathrm{p} \leq 0,05)$ para determinação da diferença estatística entre os tratamentos do rendimento e da atividade bioativa, e para atividade antifúngica, adotou-se o teste de ScottKnott, com nível de significância de 5\%. O software estatístico utilziado foi o PAST 3 (versão livre, 2019).

\section{RESULTADOS E DISCUSSÃO}

O rendimento de OE foi de $0,04 \pm 0,13$ a para $H$. stigonocarpa, e de $0,06 \pm 0,09$ a para $H$. courbaril var. courbaril. Ambos os redimentos não apresentaram diferença estatística pelo teste de Tukey $(\mathrm{p} \leq 0,05)$.

Na tabela 1, estão apresentados os resultados da atividade antioxidante dos OEs para as cascas dos frutos de $H$. stigonocarpa, $H$. courbaril var. courbaril e para o Mix (1:1) entre os OEs.

Tabela 1 - Ativiade antioxidante dos óleos essenciais das cascas dos frutos de H. stigonocarpa, H. courbaril var. courbaril e Mix (H. stigonocarpa e H. courbaril), concentração (1:1) na redução do radical livre DPPH.

\begin{tabular}{lccc}
\hline Concentração $\boldsymbol{\mu} \mathbf{L ~ m L}^{-1}$ & OEHs* $^{*}$ & OEHc**$^{* *}$ & Mix*** \\
\hline Puro & $100 \pm 0,00 \mathrm{aA}$ & $100 \pm 0,00 \mathrm{aA}$ & - \\
50 & $90,55 \pm 0,42 \mathrm{aA}$ & $100 \pm 0,00 \mathrm{aA}$ & $72,17 \pm 0,18 \mathrm{aB}$ \\
40 & $86,00 \pm 0,13 \mathrm{aB}$ & $98,63 \pm 0,16 \mathrm{aA}$ & $60,71 \pm 0,45 \mathrm{bC}$ \\
30 & $71,11 \pm 0,11 \mathrm{bB}$ & $90,69 \pm 0,33 \mathrm{bA}$ & $54,33 \pm 0,33 \mathrm{cC}$ \\
20 & $68,64 \pm 0,23 \mathrm{bcB}$ & $83,35 \pm 0,16 \mathrm{cA}$ & $20,70 \pm 0,80 \mathrm{dC}$ \\
10 & $53,05 \pm 0,69 \mathrm{~dB}$ & $71,22 \pm 0,64 \mathrm{dA}$ & $10,81 \pm 0,59 \mathrm{eC}$ \\
5 & $26,37 \pm 0,41 \mathrm{eB}$ & $58,80 \pm 0,42 \mathrm{eA}$ & - \\
2,5 & $18,94 \pm 0,63 \mathrm{eB}$ & $33,19 \pm 0,78 \mathrm{fA}$ & - \\
1,25 & - & $18,66 \pm 0,31 \mathrm{~g}$ & - \\
\hline
\end{tabular}

$* \mathrm{OEHs}=$ Óleo essencial Hymenaea stigonocarpa $. * * \mathrm{OEHc}=$ Óleo essencial Hymenaea courbaril var. courbaril. $* * * \mathrm{Mix}=(1: 1)$ dos óleos essenciais de Hymenaea stigonocarpa e H. courbaril. (-) = Não determinado. Letras pequenas iguais na mesma coluna não diferem entre si pelo teste de Tukey $(\mathrm{p} \leq 0,05)$. Letras grandes diferentes na mesma linha diferem entre si pelo teste de Tukey ( $\mathrm{p} \leq$ $0,05)$.

A atividade antioxidante na redução do DPPH para o OE H. stigonocarpa, apresentou alta eficiência, em todas as concentrações, exceto, em $1,25 \mu \mathrm{L} \mathrm{mL}^{-1}$ onde não houve inibição. A taxa de inibição foi determinada em percentagem de sequestro de DPPH, visto que, não foi utilizado modelo para determinação de $\mathrm{EC}_{50}$. Nas concentrações 40, 50 e óleo puro foram observados as maiores taxas de redução de DPPH, entretanto, não diferiram entre si pelo teste de Tukey, respectivamente. Para o OE $H$. courbaril var. courbaril, apresentou atividade antioxidante 
em todas as concentrações, em especial para as concentrações $10 ; 20 ; 30 ; 40 ; 50$ e OE puro com percentagens variando entre 71 a $100 \%$.

$\mathrm{O}$ mix de OEs $H$. stigonocarpa e $H$. courbaril var. courbaril, apresentou também eficiência como agente antioxidante, nas concentrações avaliadas. Nas concentrações onde houve atividade antioxidante, em especial para as concentrações $30 ; 40$ e $50 \mu \mathrm{L} \mathrm{mL}^{-1}$ foram obtidos resultados entre 54 a $72 \%$ de redução do DPPH.

Entre os OEs de H. stigonocarpa e H. courbaril var. courbaril na concentração de $\mathrm{OE}$ puro, não houve diferença entre ambos pelo teste de Tukey. $\mathrm{Na}$ concentração de $50 \mu \mathrm{L} \mathrm{mL} \mathrm{m}^{-1}$, apresentou diferença significativa no mix, onde o mesmo não foi observado entre os OEs, entretanto inferior ao OEs puros. Na concentração $40 \mu \mathrm{L} \mathrm{mL}^{-1}$, houve diferença estatística entre as três amostras. Nas concentrações $40 ; 30 ; 20 ; 10 ; 5 ; 2,5 \mu \mathrm{L} \mathrm{mL} \mathrm{m}^{-1}$, apresentaram diferença significativa entre as três amostras avaliadas.

Os resultados de atividade antioxidante, demonstraram que os OEs de Hymenaea stigonocarpa e Hymenaea courbaril var. courbaril, bem como, o mix apresentaram alta eficiência de inibição, sendo esta eficiência, uma nova possibilidade para novos testes em produtos farmacêuticos, na cosmetologia e na produção de alimentos. Como inexistem resultados que avaliam a atividade antioxidante do OEs das casas dos frutos de Hymenaea, a atividade antioxidante foi comparada com outros OEs de diversos grupos vegetais.

Menezes Filho et al. (2020) avaliaram os OEs das folhas e raízes de $C$. regium onde descrevem importante atividade antioxidante mesmo em baixas concentrações de OE diluído em DMSO. Os pesquisadores encontraram entre as concentrações 30,50 e $50 \mu \mathrm{L} \mathrm{mL}^{-1}$ atividade igual a $100 \%$ de redução do modelo radicalar DPPH. Da Silva et al. (2019) avaliaram a atividade antioxidante do OE de $S$. lentiscifolius onde encontraram atividade de redução de $54,4 \%$ na concentração de $100 \mathrm{mg} \mathrm{mL}^{-1}$ na redução do DPPH.

$\mathrm{Na}$ figura 1, estão apresentados resultados da porcentagem de inibição sobre o crescimento PIC para o OE da casca do fruto de $H$. stigonocarpa sobre os isolados de $S$. sclerotiorum, C. acutatum e A. flavus.

Na figura 1, observam-se alta eficiência de inibição para os isolados de S. sclerotiorum, C. acutatum e A. flavus em diferentes concentrações do OE da casca do fruto de $H$. stigonocarpa. Para $S$. sclerotiorum em especial nas maiores concentrações, apresentaram alta porcentagem de inibição de crescimento nas concentrações 12,5;25; 50 e $100 \mu \mathrm{L} \mathrm{mL}^{-1}$, com taxas entre 56 a 83\%. Entretanto, não houve diferença estatística entre as concentrações 50 e $100 \mu \mathrm{L} \mathrm{mL}^{-1}$. Para C. acutatum, também foi observado o mesmo padrão de PIC observado no isolado de $S$. sclerotiorum. Reitera-se que, as maiores concentrações entre 12,5; 25; 50 e 100 $\mu \mathrm{L} \mathrm{mL} \mathrm{L}^{-1}$, apresentaram as maiores taxas de inibição entre 66 a 75\%. Neste experimento não houve diferença significativa entre as concentrações 25 , 50 e $100 \mu \mathrm{L} \mathrm{mL}^{-1}$. Na menor concentração de 1,56 $\mu \mathrm{L} \mathrm{mL}^{-1}$ não houve atividade de inibição.

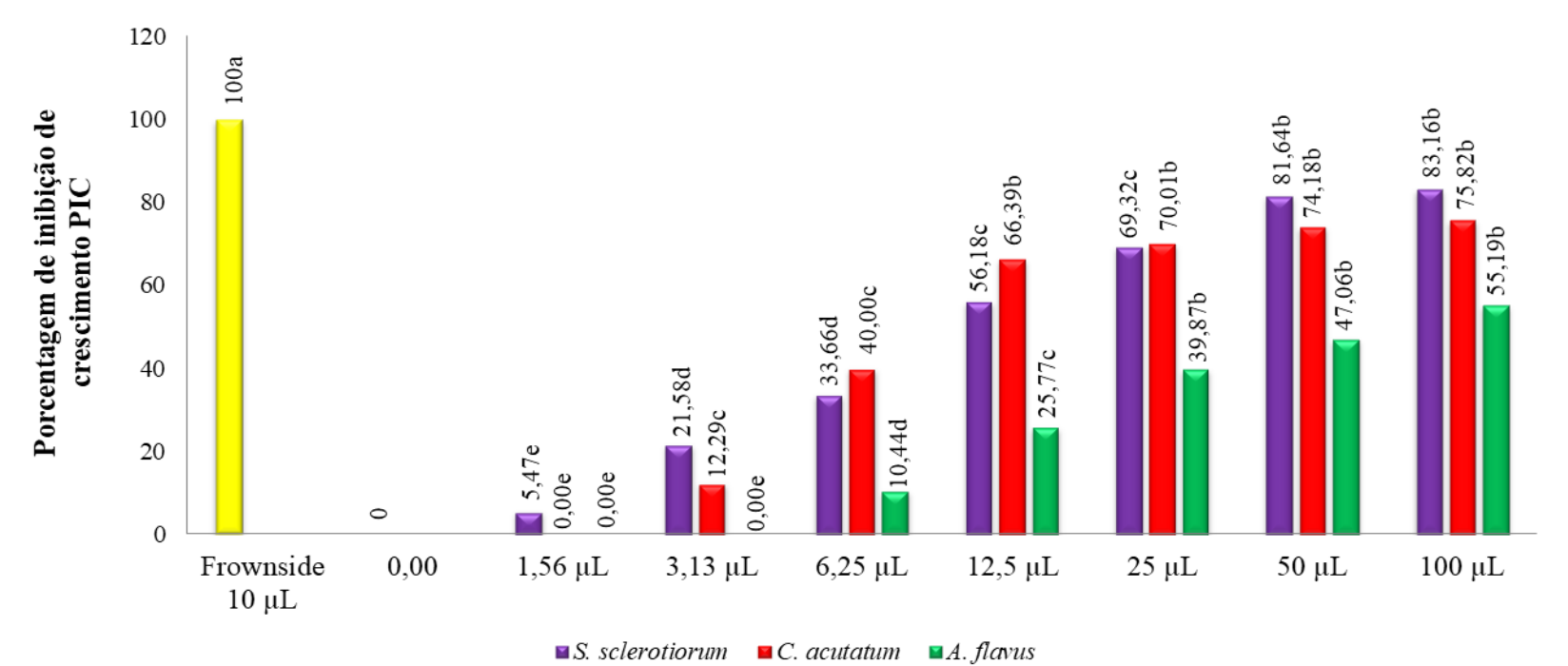

Figura 1 - Porcentagem de inibição de crescimento PIC do óleo essencial de Hymenaea stigonocarpa, sobre isolados de Sclerotinia sclerotiorum, Colletotrichum acutatum e Aspergillus flavus. Letras iguais não diferem estatisticamente pelo teste de Scott-Knott a 5\% de significância. 
O isolado de A. flavus, foi o modelo fúngico que apresentou os menores índices de atividade antifúngica, possivelmente esse fungo seja pouco sensível aos compostos do OE de H. stigonocarpa. Observa-se que a efetividade na inibição é observada apenas nas maiores concentrações 25; 50 e $100 \mu \mathrm{L} \mathrm{mL} \mathrm{mL}^{-1}$ com taxas de PIC entre 39 e $55 \%$. Entretanto, a baixa sensibilidade ao $\mathrm{OE}$ ainda apresentou bons resultados. Possivelmente a temperatura para o desenvolvimento do fungo influenciou também, entretanto negativamente nas concentrações, volatilizando os compostos de menor cadeia carbônica para monoterpenos, diterpenos e triterpenos (Benato et al., 2018; Paulus et al., 2013; Radünz et al., 2006).

Os três ensaios fúngicos foram comparados ao fungicida comercial Frowncide na concentração de $10 \mu \mathrm{L} \mathrm{mL}^{-1}$ com $100 \%$ de PIC.

Silva et al. (2018) avaliaram o OE de P. guaja$v a$ (goiaba) em duas épocas de extração, onde obtiveram inibição micelial para $S$. sclerotiorum de 94,9 e de $93,4 \%$ na concentração de $300 \mu \mathrm{L}$ $\mathrm{mL}^{-1}$, de 90,8 e $90,0 \%$ na concentração de $200 \mu \mathrm{L}$ $\mathrm{mL}^{-1}$ e de 77,5 e $80,0 \%$ na menor concentração usual de $100 \mu \mathrm{L} \mathrm{mL}^{-1}$. Lima et al. (2014) verificaram inibição entre 95 a $100 \%$ no crescimento em A. flavus proporcionado pelo $\mathrm{OE}$ de cravo-daíndia (S. aromaticum). Já para o OE de melaleuca, os pesquisadores obtiveram PIC em todas as concentrações avaliadas, com maior eficiência na concentração de $15 \mu \mathrm{L}$ com $8 \%$ de inibição. Nos estudos desenvolvidos por Vivas et al. (2006), os pesquisadores verificaram que o efeito fungicida do OE do capim-santo inibiu completamente o crescimento micelial em $C$. acutatum em concentrações superiores a $100 \mu \mathrm{L} \mathrm{mL}^{-1}$.

Na figura 2, estão apresentadas as porcentagens de inibição de crescimento pelo $\mathrm{OE}$ da casca do fruto de Hymenaea courbaril var. courbaril sobre os isolados de $S$. sclerotiorum, $C$. acutatum e $A$. flavus em diferentes concentrações.

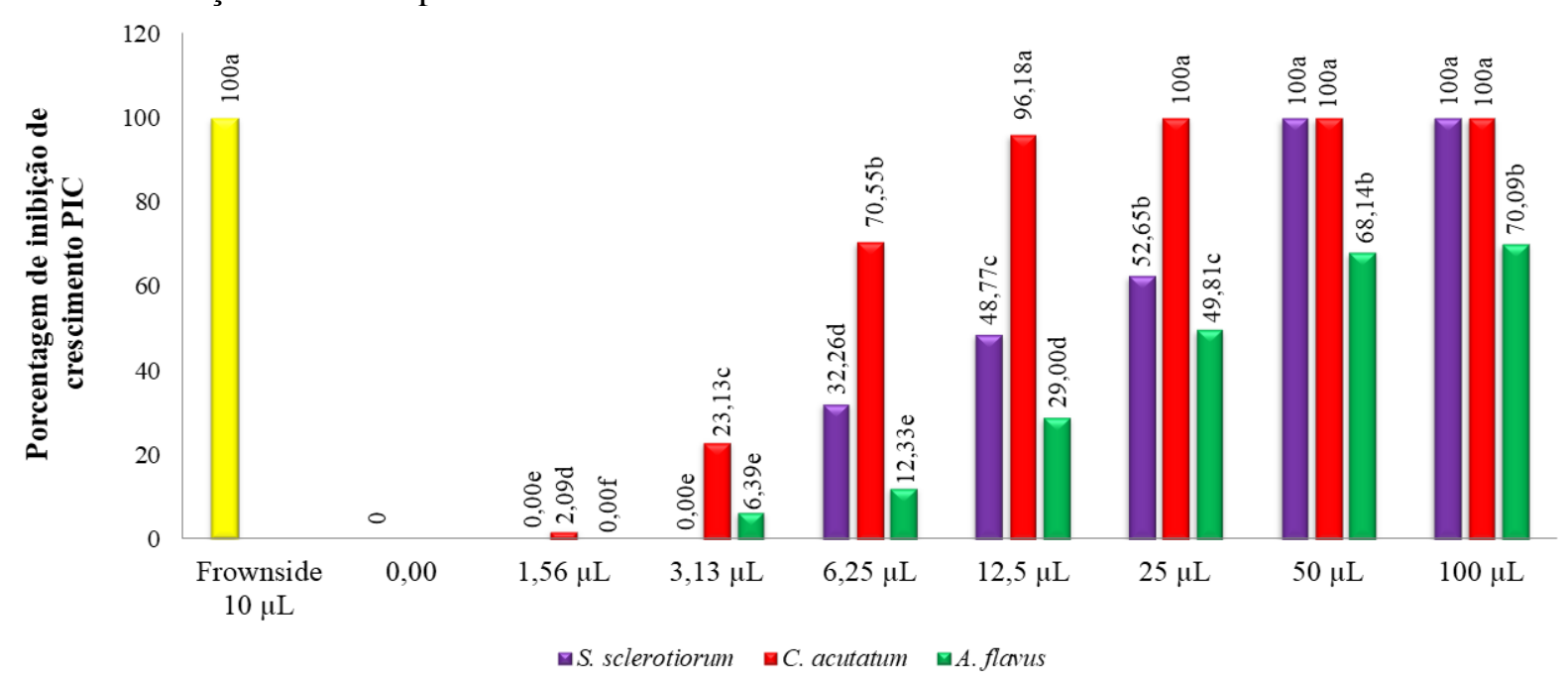

Figura 2 - Porcentagem de inibição de crescimento PIC do óleo essencial de Hymenaea courbaril var. courbaril, sobre isolados de Sclerotinia sclerotiorum, Colletotrichum acutatum e Aspergillus flavus. Letras iguais não diferem estatisticamente pelo teste de Scott-Knott a 5\% de significância.

O OE de H. courbaril var. courbaril também apresentou boa eficiência de PIC sobre os isolados de $S$. sclerotiorum e $C$. acutatum. Em todas as concentrações, exceto para 1,56 e $3,13 \mu \mathrm{L} \mathrm{mL}^{-1}$. Foi observado que ambos os isolados são sensíveis a complexa constituição do $\mathrm{OE}$ da casca do fruto de $H$. courbaril. Para A. flavus foi observado bons resultados, entretanto, como observado para o $\mathrm{OE}$ de $H$. stigonocarpa, o fungo apresenta também resistência em todas as concentrações para este OE.

As melhores concentrações usuais para inibir o desenvolvimento de $S$. sclerotiorum, $C$. acutatum e A. flavus são concentrações acima de $12,5 \mu \mathrm{L}$ $\mathrm{mL}^{-1}$, que apresentaram PIC comparável ao fungicida de referência. Entretanto, para A. flavus as concentrações 50 e $100 \mu \mathrm{L} \mathrm{mL}^{-1}$ apresentaram ser as mais eficazes para inibição fúngica.

Bonaldo et al. (2007), encontraram para o OE de E. citriodora, atividade de inibição micelial entre 90 a 100\% para S. rolfsii, Phytophthora sp., A. alternata, $C$. sublineolum e $R$. solani em concentrações entre 40 a $1000 \mu \mathrm{L}$. Schwan-Estrada et al. (2000), encontraram em seus estudos in vitro, para o extrato bruto e $\mathrm{OE}$ de manjerona boa eficiência de inibição de crescimento micelial, e de 
esporulação em diversos fungos fitopatogênicos testados.

$\mathrm{Na}$ figura 3, estão apresentados os resultados da porcentagem de inibição de crescimento pelo mix de OEs das cascas de ambos os frutos de H. stigonocarpa e $H$. courbaril var. courbaril sobre isolados de S. sclerotiorum, C. gloeosporioides e A. niger em diferentes concentrações.

$\mathrm{O}$ mix dos OEs demonstrou ser mais eficaz apenas para o isolado de $S$. sclerotiorum. Para os isolados de C. gloeosporioides foi observado uma discreta PIC, e com menor intencidade de inibição, seguido para A. niger. O S. sclerotiorum

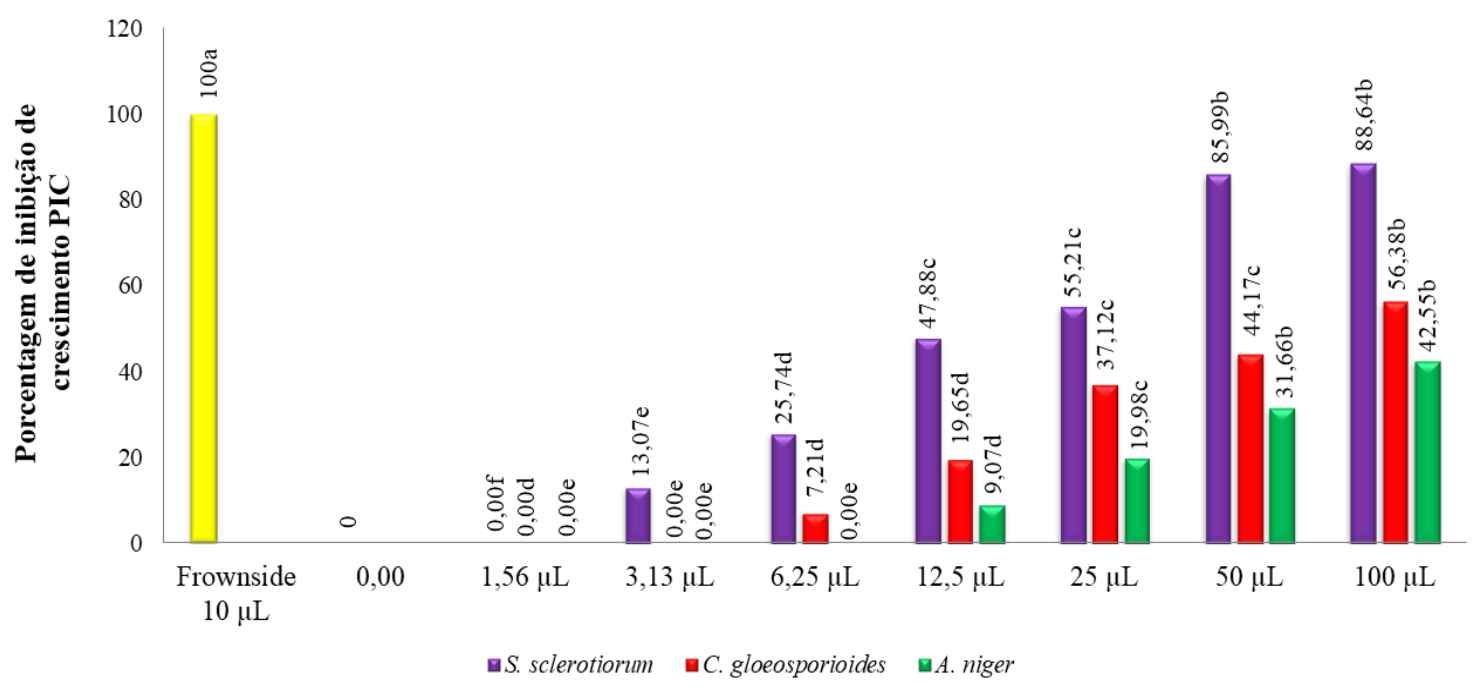

Figura 3 - Porcentagem de inibição de crescimento PIC do mix dos óleos essenciais de Hymenaea stigonocarpa e Hymenaea courbaril var. courbaril, sobre isolados de Sclerotinia sclerotiorum, Colletotrichum gloeosporioides e Aspergillus niger. Letras iguais não diferem estatisticamente pelo teste de Scott-Knott a 5\% de significância.

Lima et al. (2014), encontraram baixa eficiência no controle de crescimento micelial em A. niger utilizando OE de Melaleuca alternifolia. Os pesquisadores obtiveram inibição de 1,3 e $14 \%$ para as concentrações 5,10 e $15 \mu \mathrm{L}$. Souza Júnior et al. (2009), avaliaram diferentes concentrações de OEs de alecrim-pimenta, alfavaca-cravo, capim-santo e cidrão onde obtiveram $100 \%$ de inibição de crescimento em todas as concentrações avaliadas, e entre 44,0 a $69,0 \%$ para o OE das folhas de goiaba. Carnelossi et al. (2009), e Silva et al. (2009), encontraram inibição de $100 \%$ para C. gloeosporioides utilizando $\mathrm{OE}$ de capim-limão (Cymbopogon citratus) em concentrações entre 10 e $100 \mu \mathrm{L}$. Celoto et al. (2008), avaliaram diferentes extratos vegetais (aquoso e hidroetanólico) onde encontraram também importantes resultados de PIC para C. gloeosporioides. $\mathrm{O} \mathrm{OE}$ de $C$. zeylanicum apresentou boa eficiência de inibição para A. niger nos estudos de Pawar e Thaker (2006). demonstra ser altamente sensível, corroborando com os resultados apresentados nas (Figuras 1 e 2) para OEs puros. Na figura 3 , observa-se que o doseamento de ambos os OEs das cascas dos frutos de Hymenaea não apresentou resultados superiores quando comparado aos $\mathrm{OE}$ puros. $\mathrm{O}$ mesmo é observado quando comparado ao fungicida de referência, com resultados inferiores conforme observado no teste de Scott-Knott a 5\%.

O intuíto da avaliação pelo mix de OEs foi para observar possível potencialização de ambos os OEs, entretanto, não demonstraram resultados satisfatórios para os isolados investigados.

\section{CONCLUSÃO}

Os óleos essenciais de Hymenaea stigonocarpa e Hymenaea courbaril var. courbaril apresentaram bons rendimentos de extração, bem como alta eficiência biológica como agentes antioxidante (puro e mix (1:1)) e antifúngico in vitro. Os isolados de Sclerotinia slcerotiorum, Colletotrichum acutatum e Aspergillus flavus demonstraram ser altamente senssíveis aos óleos essenciais quando aplicados individualmente, e o mesmo foi observado apenas para Sclerotinia sclerotiorum no mix (1:1) dos óleos essenciais para ambas as espécies do gênero Hymenaea.

Trabalhos futuros deverão ser realizados avaliando as atividades antioxidante e antifúgica em modelos biológicos para determinação da eficácia como possíveis novos agentes antioxidantes em alimentos e formulações farmacêuticas, e também como em nanocápsulas e microcápsulas contendo óleo essencial verificando 
o comportamento dos óleos essenciais em casa-devegetação, ou in situ em campos de cultivos.

\section{AGRADECIMENTOS}

Ao Instituto Federal Goiano, Campus Rio Verde; aos laboratórios de Química Tecnológica, Bioensaios e Biomoléculas, Química de Produtos Naturais, Fisiologia Vegetal e Sistemática Vegetal; ao Herbário do IF Goiano, Campus Rio Verde; ao órgãos de fomento em pesquisa, $\mathrm{CNPq}$, CAPES, FINEP, e a FAPEG pela bolsa de mestrado em Agroquímica a Antonio Carlos Pereira de Menezes Filho.

\section{REFERÊNCIAS BIBLIOGRÁFICAS}

Amirghofran Z, Azadbakht M, Karii MH. Evaluation of the immunomodulatory effects of five herbal plants. Journal of Ethnopharmacology, v.72, n.1-2, p.167-172, 2000. https://doi.org/10.1016/S0378-8741(00)00234-8

Angle JS, Dunn KA, Wagner GH. Effect of cultural practices on the soil populations of Aspergillus flavus and Aspergillus parasiticus. Soil Science Society of America Journal, v.46, n.2, p.301-303, 1982. https://doi.org/10.2136/sssaj1982.03615995004600020017 $\mathrm{x}$

Aquino FG, Ribeiro JF, Walter BMT. Espécies vegetais de uso múltiplo em reservas legais de Cerrado, Balsas/MA. Revista Brasileira de Biociências, v.5, n.1, p.147-149, 2007.

https://doi.org/10.1590/0100-5405/175659

Athié I, Castro MFPM, Gomes RAR, Valentini SRT. Conservação de grãos. Campinas SP. Fundação Cargill. 236p. 1998.

Bagherabadi S, Zafari D, Anvar FG, Damm U. Colletotrichum gloeosporioides s. str., the causal agent of a leaf spot disease of Schefflera arboricola in Iran. Mycologia Iranica, v.5, n.1, p.29-34, 2018. https://doi.org/ 10.22043/mi.2019.118404

Benato EA, Belletti TC, Terao D, Franco DAS. Óleos essenciais e tratamento térmico no controle pós-colheita de bolor verde em laranja. Summa Phytopathologica, v.44, n.1, p.65-71, 2018.

https://doi.org/10.22043/MI.2019.118404

Bizzo HR, Hovell AMC, Rezende CM. Óleos essenciais no Brasil: Aspectos gerais, desenvolvimento e perspectivas. Química Nova, v.32, n.3, p.588-594, 2009. https://doi.org/10.1590/S0100-40422009000300005

Boland GJ, Hall R. Index of plant hosts of Sclerotinia sclerotiorum. Canadian Journal Plant Pathology, v.16, n.2, p.93108, 1994.

https://doi.org/10.1080/07060669409500766

Bonaldo SM, Schwan-Estrada KRF, Stangarlin JR, Cruz MÊS, Fiori-Tutida ACG. Contribuição ao estudo das atividades antifúngica e elicitora de fitoalexinas em sorgo e soja por eucalipto (Eucalyptus citriodora). Summa Phytopa- thologica, v.33, n.4, p.383-387, 2007.

https://doi.org/10.1590/S0100-54052007000400011

Botelho AS, Ferreira RA, Malavasi MdeM, Davide AC. Aspectos morfológicos de frutos, sementes, plântulas e mudas de jatobá-do-cerrado (Hymenaea stigonocarpa Mart. ex Hayne) - Fabaceae. Revista Brasileira de Sementes, v.22, n.1, p.144-152, 2000.

http://dx.doi.org/10.1590/S0101-31222011000200004

Bragança CAD, Damma U, Baroncelli R, Massola Júnior NS, Crous PW. Species of the Colletotrichum acutatum complex associated with anthracnose diseases of fruit in Brazil. Fungal Biology, v.120, n.4, p.547-561, 2016. https://doi.org/10.1016/j.funbio.2016.01.011

Carnelossi PR, Schwan-Estrada KRF, Cruz MÊS, Itako AT, Mesquini RM. Óleos essenciais no controle pós-colheita de Colletotrichum gloeosporioides em mamão. Revista Brasileira de Plantas Medicinais, v.11, n.4, p.399-406, 2009. https://doi.org/10.1590/S1516-05722009000400007

Carvalho Filho JLS, Arrigoni-Blank MF, Blank AF, Rangel MAS. Produção de mudas de jatobá (Hymenaea courbaril L.) em diferentes ambientes, recipientes e composições de substratos. Cerne, v.9, n.1, p.109-118, 2003. https://ri.ufs.br/handle/riufs/1590

Celoto MIB, Papa MFS, Sacramento LVS, Celoto FJ. Atividade antifúngica de extratos de plantas a Colletotrichum gloeosporioides. Acta Scientiarum, v.30, n.1, p.1-5, 2008. https://doi.org/10.4025/actasciagron.v30i1.1104

Da Silva ER, Pawlowski Â, Lazarotto DC, Soares GLG. Antioxidant activity of essential oils from leaves of Schinus lentiscifolius Marchand. Revista Cubana de Plantas Medicinales, v.24, n.2, p.783, 2019.

Da Silva SM, Pereira AL, García-Zapata MTA. Manifestações bucais na infecção pelo vírus da imunodeficiência humana: uma revisão sistemática da literatura. Revista da Faculdade de Odontologia de Porto Alegre, v.52, n.1/3, p.57-65, 2011. https://doi.org/10.22456/2177-0018.30650

De Candolle AP. The leguminous seed. Phytomorphology, v.1, p.117-150, 1825.

De Souza PF, Da Silva GH, Henriques ÍGN, Campelo GJ, Alves GS. Atividade antifúngica de diferentes concentrações de extrato de alho em sementes de ingá (Inga edulis). Revista Verde de Agroecologia e Desenvolvimento Sustentável, v.5, n.5, p.8-13, 2010.

Deshmukh H, Rambach G, Sheooard DC, Lee M, Hagleitner M, Hermann M, Würzner R, Lass-Flörl C, Speth C. Galactosaminogalactan secreted from Aspergilus fumigatus and Aspergillus flavus induces platelet activation. Microbes and Infection, 2020. https://doi.org/10.1016/j.micinf.2019.12.004

Deus RJA, Alves CN, Arruda MSP. Avaliação do efeito antifúngico do óleo resina e do óleo essencial de copaíba (Copaifera multijuga Hayne). Revista Brasileira de Plantas Medicinais, v.13, n.1, p.1-7, 2011.

https://doi.org/10.1590/S1516-05722011000100001

Dhingra OD, Coelho Neto RA. Micotoxinas em grãos. Revisão Anual de Patologia de Plantas, v.6, n.1, p.49-101, 1998. 
Fallah S, Mouguee S, Rostaei M, Adavi Z, Lorigooini Z. Chemical compositions and antioxidant activity of essential oil of wild and cultivated Dracocephalum kotshyi frown in different ecosystems: A comparative study. Industrial Crops and Products, v.143, p.111885, 2020. https://doi.org/10.1016/j.indcrop.2019.111885

Fawcett CH, Spencer DM. Plant chemotherapy with natural products. Annual Review of Phytopathology, v.8, n.1, p.403-418, 1970.

Filho RML, Oliveira SM, Menezes M. Caracterização enzimática e patogenicidade cruzada de Colletotrichum spp. associados a doenças de pós-colheita. Fitopatologia Brasileira, v.28, n.6, p.620-625, 2003. http://dx.doi.org/10.1590/S0100-41582003000600007

Garcia RÁ, Juliatti FC, Barbosa KAG, Cassemiro TA. Atividade antifúngica de óleo e extratos vegetais sobre Sclerotinia sclerotiorum. Bioscience Journal, v.28, n.1, p.48-57, 2012.

Golshani S, Karamkhani F, Monsef-Esfehani HR, Abdollahi M. Antinociceptive effects of the essential oil of Dracocephalum kotschyi in the mouse writhing test. Journal of Pharmacy \& Pharmaceutical Sciences, v.98, n.7, p.76-79, 2004.

Hamid MR, Xie J, Wu S, Maria SK, Zheng D, Hamidou AA, Wang Q, Cheng J, Fu Y, Jiang D. A novel deltaflexivirus that infect the plant fungal pathogen, Sclerotinia sclerotiorum, can be transmited among host vegetative incompatible strains. Viruses, v.10, n.295, p.1-15, 2018.

https://doi.org/10.3390/v10060295

Himed L, Merniz S, Monteagudo-Olivan R, Barkat M, Coronas J. Antioxidant activity of the essential oil of Citrus limon before and after its encapsulation in amorphous $\mathrm{SiO}^{2}$. Scientific African, v.6, p.181, 2019. https://doi.org/10.1016/j.sciaf.2019.e00181

Hüller AS, Santini EJ, Da Silveira AG, Valcorte G, De Oliveira LH, Estigarribia MG. Capacidade antifúngica do extrato vegetal de Ruta graveolens sobre o desenvolvimento dos fungos apodrecedores Gloeophylum trabeum e Pycnoporus sanguineus. Revista Brasileira de Iniciação Cientifica, v.6, n.6, p.31-43, 2019.

Jahaniani F, Ebrahimi AS, Rahbar-Roshandel N, Mahmoudian M. Xanthomicrol is the main cytotoxic component of Dracocephalum kotschyi and a potential anti-cancer agent. Phytochemistry, v.66, n.13, p.1581-1592, 2005. https://doi.org/10.1016/j.phytochem.2005.04.035

Lee YT, Langeheim JH. Systematics of the genus Hymenaea L. (Leguminosae; Detaroeae). University of California Publications in Botany, v.69, p.1-109, 1975.

Lima A, Ribeiro AS, Bonaldo SM. Efeito dos óleos essenciais de Syzygium aromaticum e Melaleuca alternifolia sobre isolados de Aspergillus sp. Scientific Eletronic Archives, v.5, p.63-67, 2014.

Lima HC, Pinto RB. Hymenaea. In: Lista de espécies da flora do Brasil. Jardim Botânico do Rio de Janeiro. Disponível em http://floradobrasil.jbrj.gov.br/floradobrasil/FB22971. Acesso em 25 mai. 2020.

Lima IO, Oliveira RAG, Lima EO, Farias NMP, De Souza EL. Atividade antifúngica de óleos essenciais sobre espécies de Candida. Revista Brasileira de Farmacognosia, v.
16, n. 2, p. 197-201, 2006.

https://doi.org/10.1590/S0102-695X2006000200011

Lopes AJ, Jansen U, Capone D, Jansen JM. Aspergiloses pulmonares. Pulmão, v.13, n.1, p.34-44, 2004.

Mackinder B. Detarieae. In: Lewis G, Schrire B, MacKinder B, Lock M. (eds.), Legumes of the world. The Royal Botanical Gardens, Kew, p.69-71, 2005.

Magalhães N, Cavalcante AV, Andrade LS, Wanderley CRP, Marinho G, Pessoa KAR. Produção de ácido cítrico por Aspergillus niger NA 400 a partir de resíduo agroindustrial. Engenharia Sanitária e Ambiental, v.24, n.1, p.101-107, 2019.

https://doi.org/10.1590/s1413-41522019167153

Menezes Filho ACP, Christofoli M, De Sousa WC, Castro CFS. Atividades antioxidante e antifúngica dos óleos essenciais de Cochlospermum regium frente à Sclerotinia sclerotiorum e Colletotrichum gloeosporioides. Colloquium Agrariae, v.16, n.1, p.109-116, 2020. https://doi.org/10.5747/ca.2020.v16.n1.a35

Menezes Filho ACP, Oliveira Filho, JG, Deminski GO, Jesus AP, De Andrade MSB, Castro CFS. Avaliação colorimétrica e caracterização morfológica por microscopia óptica de alta resolução das farinhas dos frutos do jatobá, jambolão e seriguela. Multi-Science Journal, v.2, n.1, p.16-22, 2019.

https://doi.org/10.33837/msj.v2i1.544

Mezza GN, Borganello AV, Grosso NR, Fernandez H, Pramparo MC. Antioxidant activity of rosemary essential oil fractions obtained by molecular distillation and their on oxidative stability of sunflower oil. Food Chemistry, v.242, p.9-15,2018.

https:// doi.org/10.1016/j.foodchem.2017.09.042

Paulus D, Valmorbida R, Toffoli E, Nava GA. Teor e composição química de óleo essencial de cidró em função da sazonalidade e horário de colheita. Horticultura Brasileira, v.31, n.2, p. 203-209, 2013. https://doi.org/10.1590/S0102-05362013000200005

Pawar VC, Thaker VS. In vitro efficacy of 75 essential oils against Aspergillus niger. Mycoses, v.49, n.4, p.316-323, 2006.

https://doi.org/10.1111/j.1439-0507.2006.01241.x

Pestana LTC. Estudo taxonômico de Hymenaea L.: complexo H. courbaril, H. martiana e H. stigonocarpa (Fabaceae: Caesalpinioideae: Detarieae). Dissertação de Mestrado. Fundação Universidade Federal de Mato Grosso do Sul.

Putrick TC, Costa MLN, Theodoro GF. Efeito de fertilizante foliar sobre isolados de Sclerotinia sclerotiorum. Revista de Agricultura Neotropical, v.5, n.2, p.83-90, 2018. https://doi.org/10.32404/rean.v5i2.1453

Radünz LL, Melo EC, Barbosa LCA, Silva Santos RH, Barbosa FF, Martinazzo AP. Influência da temperatura do ar de secagem no rendimento do óleo essencial de hortelãcomum (Mentha x villosa Huds). Engenharia na Agricultura, v.14, n.4, p.250-257, 2006.

Ramos K, Andreani Júnior R, Kozusny-Andreani DI. Óleos essenciais e vegetais no controle in vitro de Colletotrichum gloeosporioides. Revista Brasileira de Plantas Medicinais, v.18, n.2, supl.I, p.605-612, 2016. https://doi.org/10.1590/1983-084x/15_192 
Roesler R, Malta LG, Carrasco LC, Holanda RB, Sousa CAS, Pastore GM. Atividade antioxidante de frutas do cerrado. Ciência e Tecnologia de Alimentos, v.27, n.1, p.5360, 2007.

http:// doi.org /10.1590/S0101-20612007000100010

Sajjadi SE, Atar AM, Yektaian A. Antihyperlipidemic effect of hydroalcoholic extract, and polyphenolic fraction from Dracocephalum kotschyi Boiss. Pharmaceutica Acta Helvetae, v.73, n.3, p.167-170, 1998.

https://doi.org/10.1016/S0031-6865(98)00016-8

Schwan-Estrada KRF, Stangarlin JR, Cruz MES. Uso de plantas medicinais no controle de doenças de plantas. Uso de extratos vegetais no controle de fungos fitopatogênicos. Revista Floresta, v.30, n.1, p.129-137, 2000.

Shami NJIE, Moreira EAM. Licopeno como agente antioxidante. Revista de Nutrição, v.17, n.2, p.227-236, 2004. http:// doi.org/10.1590/S1415-52732004000200009

Silva AC, Sales NLP, De Araújo AC, Júnior CFC Efeito in vitro de compostos de plantas sobre o fungo Colletotrichum gloeosporioides Penz. isolado do maracujazeiro. Ciência Agrotecnologia, v.33, Edição Especial, p.18531860, 2009. http:// doi.org/10.1590/S1413-70542009000700026

Silva AC, Sales NLP, De Araújo AV, Júnior CFC. Efeito in vitro de compostos de plantas sobre o fungo Colletotrichum gloeosporioides Penz. isolado do maracujazeiro. Ciência e Agrotecnologia, v. 33, Edição especial, p.18531860, 2009.

http:// doi.org/10.1590/S1413-70542009000700026

Silva EAJ, Da Silva CP, Alves CCF, Alves JM, Souchie EL, Barbosa LCA. Chemical composition of the essential oil of Psidium guajava leaves and its toxicity against Sclerotinia sclerotiorum. Semina: Ciências Agrárias, v. 39, n. 2, p. 865-874, 2018

http:// doi.org/10.5433/1679-0359.2018v39n2p865

Silva MR, Silva MAAP, Chang YK. Utilização da farinha de jatobá (Hymenaea stigonocarpa Mart.) na elaboração de biscoitos tipo cookie e avaliação de aceitação por testes sensoriais afetivos univariados e multivariados. Ciência e Tecnologia de Alimentos, v.18, n.1, p.25-34, 1998. http:// doi.org/10.1590/S0101-20611998000100007

Souza IM, Funch LS, De Queiroz LP. Flora da Bahia: Leguminosae - Hymenaea (Caesalpinioideae: Detarieae). Sitientibus série Ciências Biológicas, v.16, 2016. http:// doi.org/10.13102/scb1092

Souza IM. O gênero Hymenaea L. (Leguminosae: Caesalpinioideae) para o estado da Bahia: aspectos sobre a taxonomia, fenologia e biologia floral. Dissertação de Mestrado. Universidade Estadual de Feira de Santana, 2013.

Souza Júnior ITS, Sales NLP, Martins ER. Efeito fungitóxico de óleos essenciais sobre Colletotrichum gloeosporioides, isolado do maracujazeiro amarelo. Biotemas, v.22, n.3, p.77-83, 2009.

https://doi.org/10.5007/2175-7925.2009v22n3p77

Tico BM, Da Silva HF, Da Silva EC, Silva GR, Do Nascimento LC. Óleos essenciais no controle do Fusarium sp. da cana de açúcar in vitro. Revista Brasileira de Meio Ambiente, v.7, n.3, p.070-079, 2019.

https://doi.org/10.5281/zenodo.3595058
Vivas M, Silva DG, Costa H, Silveira SF, Pereira AJ. Inibição in vitro do crescimento micelial de Colletotrichum acutatum por extrato bruto aquoso e óleo essencial de Cymbopogon citratus (DC) Stapf e Eucalyptus citriodora Hooker. Fitopatologia Brasileira, v.31, 2006. 